\title{
An Energy-Efficient MAC Protocol for Wireless Wearable Computer Systems
}

\author{
Jounghoon Beh ${ }^{1 *}$, Kyeong Hur ${ }^{2}$, Wooil Kim ${ }^{3}$, and Yang-Ick Joo ${ }^{4}$, Member, KIICE \\ ${ }^{1}$ Instutute for Advanced Computer Studies, University of Maryland, College Park, MD 20742, USA \\ ${ }^{2}$ Department of Computer Education, Gyeongin National University of Education, Anyang 430-040, Korea \\ ${ }^{3}$ School of Computer Science and Engineering, University of Incheon, Incheon 406-772, Korea \\ ${ }^{4}$ Division of Electrical and Electronics Engineering, Korea Maritime University, Busan 606-791, Korea
}

\begin{abstract}
Wearable computer systems use the wireless universal serial bus (WUSB), which refers to USB technology that is merged with WiMedia physical layer and medium access control layer (PHY/MAC) technical specifications. WUSB can be applied to wireless personal area network (WPAN) applications as well as wired USB applications such as PAN. WUSB specifications have defined high-speed connections between a WUSB host and WUSB devices for compatibility with USB 2.0 specifications. In this paper, we focus on an integrated system with a WUSB over an IEEE 802.15.6 wireless body area network (WBAN) for wireless wearable computer systems. Due to the portable and wearable nature of wearable computer systems, the WUSB over IEEE 802.15.6 hierarchical medium access control (MAC) protocol has to support power saving operations and integrate WUSB transactions with WBAN traffic efficiently. In this paper, we propose a low-power hibernation technique (LHT) for WUSB over IEEE 802.15.6 hierarchical MAC to improve its energy efficiency. Simulation results show that the LHT also integrates WUSB transactions and WBAN traffic efficiently while it achieves high energy efficiency.
\end{abstract}

Index Terms: Hierarchical MAC, Wireless body area networks, Wearable computer, Wireless USB

\section{INTRODUCTION}

A recent major development in computer technology is the advent of the wearable computer system, which is based on human-centric interface technology trends and ubiquitous computing environments [1, 2]. Wearable computer systems use the wireless universal serial bus (WUSB), which refers to USB technology that is merged with WiMedia physical layer and medium access control layer (PHY/MAC) technical specifications. WUSB can be applied to wireless personal area network (WPAN) applications as well as wired USB applications such as PAN. WUSB specifications have defined high-speed connections between a WUSB host and WUSB devices for compatibility with USB 2.0 specifications [3, 4].

A wireless body area network (WBAN), which describes the application of wearable computing devices, allows the integration of intelligent, miniaturized, low-power, invasive/ on-invasive sensor nodes that monitor body functions and the surrounding environment. Each intelligent node has sufficient capability to process and forward information to a base station for diagnosis and prescription [5].

Received 24 October 2012, Revised 16 December 2012, Accepted 03 January 2013

*Corresponding Author Jounghoon Beh (E-mail: jhbeh@umiacs.umd.edu, Tel: +1-301-405-2876)

Instutute for Advanced Computer Studies, University of Maryland, 7965 Baltimore Avenue, College Park, MD 20742, USA. 

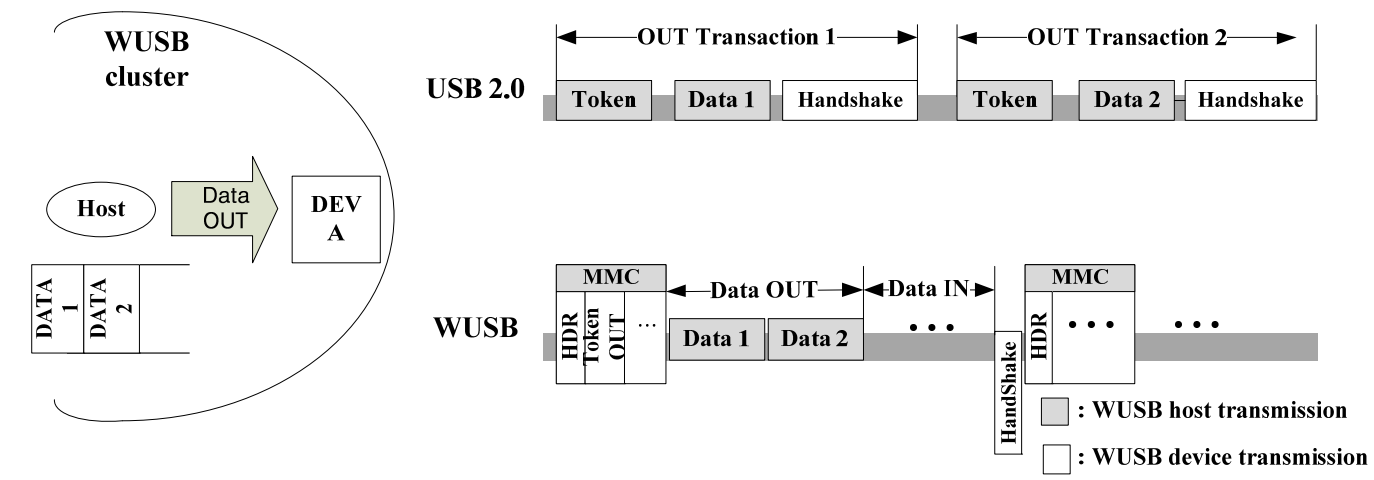

Fig. 1. Relationship between USB transaction and WUSB transaction. USB: universal serial bus, WUSB: wireless USB, MMC: micro-scheduled management command.

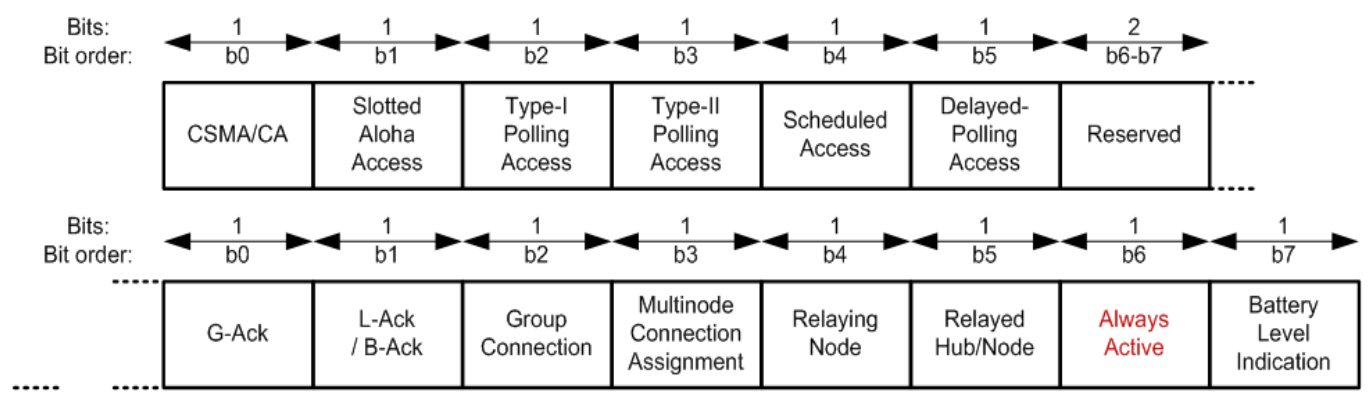

Fig. 2. Wireless body area network (WBAN) medium access control (MAC) capability format standard. CSMA/CA: carrier sense multiple access/collision avoidance.

\begin{tabular}{|c|c|c|c|c|c|c|c|}
\hline \multirow[t]{2}{*}{$\begin{array}{l}\text { Octets: } \\
\text { Octet order: }\end{array}$} & $\begin{array}{c}6 \\
0-5\end{array}$ & $\frac{6}{0-5}$ & $\frac{6}{0-5}$ & $\frac{2}{0-1}$ & $\frac{1}{0}$ & $\frac{1}{0}$ & \\
\hline & $\begin{array}{l}\text { Recipient } \\
\text { Address }\end{array}$ & $\begin{array}{l}\text { Sender } \\
\text { Address }\end{array}$ & $\begin{array}{l}\text { Former } \\
\text { Hub } \\
\text { Address }\end{array}$ & $\begin{array}{c}\text { MAC } \\
\text { Capability }\end{array}$ & $\begin{array}{c}\text { PHY } \\
\text { Capability }\end{array}$ & $\begin{array}{l}\text { Connection } \\
\text { Change } \\
\text { Indicator }\end{array}$ & \\
\hline \multirow[t]{2}{*}{$\begin{array}{l}\text { Octets: } \\
\text { Octet order: }\end{array}$} & $\frac{2}{0-1}$ & $\frac{2}{0-1}$ & $\begin{array}{l}\geq 7 \\
z-R\end{array}$ & $\begin{array}{l}\geq 7 \\
L-R\end{array}$ & $\begin{array}{l}\geq 7 \\
\mathrm{~L}-\mathrm{R}\end{array}$ & $\begin{array}{l}\geq 8 \\
\mathrm{~L}-\mathrm{R}\end{array}$ & $\begin{array}{c}3 \\
\mathrm{~L}-\mathrm{R}\end{array}$ \\
\hline & $\begin{array}{l}\text { Wakeup } \\
\text { Phase }\end{array}$ & $\begin{array}{l}\text { Wakeup } \\
\text { Period }\end{array}$ & $\begin{array}{c}\text { Explict/Impl } \\
\text { Uplink } \\
\text { Request }\end{array}$ & $\begin{array}{l}\text { Explicit/Implic } \\
\text { Downlink } \\
\text { Request IE }\end{array}$ & $\begin{array}{l}\text { Explicit/Implic } \\
\text { Bilink } \\
\text { Request IE }\end{array}$ & $\begin{array}{l}\text { Explicit/Implici } \\
\text { Delayed Bilink } \\
\text { Request IE }\end{array}$ & $\begin{array}{c}\text { Group } \\
\text { Connection } \\
\text { IE }\end{array}$ \\
\hline
\end{tabular}

Fig. 3. Connection request control frame format. MAC: medium access control.

The WUSB channel is a continuous sequence of linked application-specific control packets, called micro-scheduled management commands (MMCs). As shown in Fig. 1, WUSB maps the USB 2.0 transaction protocol onto the TDMA micro-scheduling feature (device receive, DR; device transmit, DT; device notification time slots, DNTS). MMCs are used to advertise WUSB channel time allocations for point-to-point data communications between the host and the endpoints of the devices in the WUSB cluster. In this paper, we propose a low-power hibernation technique (LHT) for WUSB over IEEE 802.15.6 hierarchical MAC to improve its energy efficiency.

\section{LHT FOR WUSB OVER WBAN ARCHITECTURE}

WBAN slave devices that have received beacons from the WBAN host schedule their receiving and transmitting operations according to information delivered by the beacon. The IEEE 802.15.6 WBAN superframe begins with a beacon period in which the WBAN hub performing the WUSB host's role (WUSB/WBAN host) sends the beacon. The data transmission period in each superframe is classified into the exclusive access phase 1 (EAP1), random access phase 1 (RAP1), Type-I/II access phase, EAP2, 


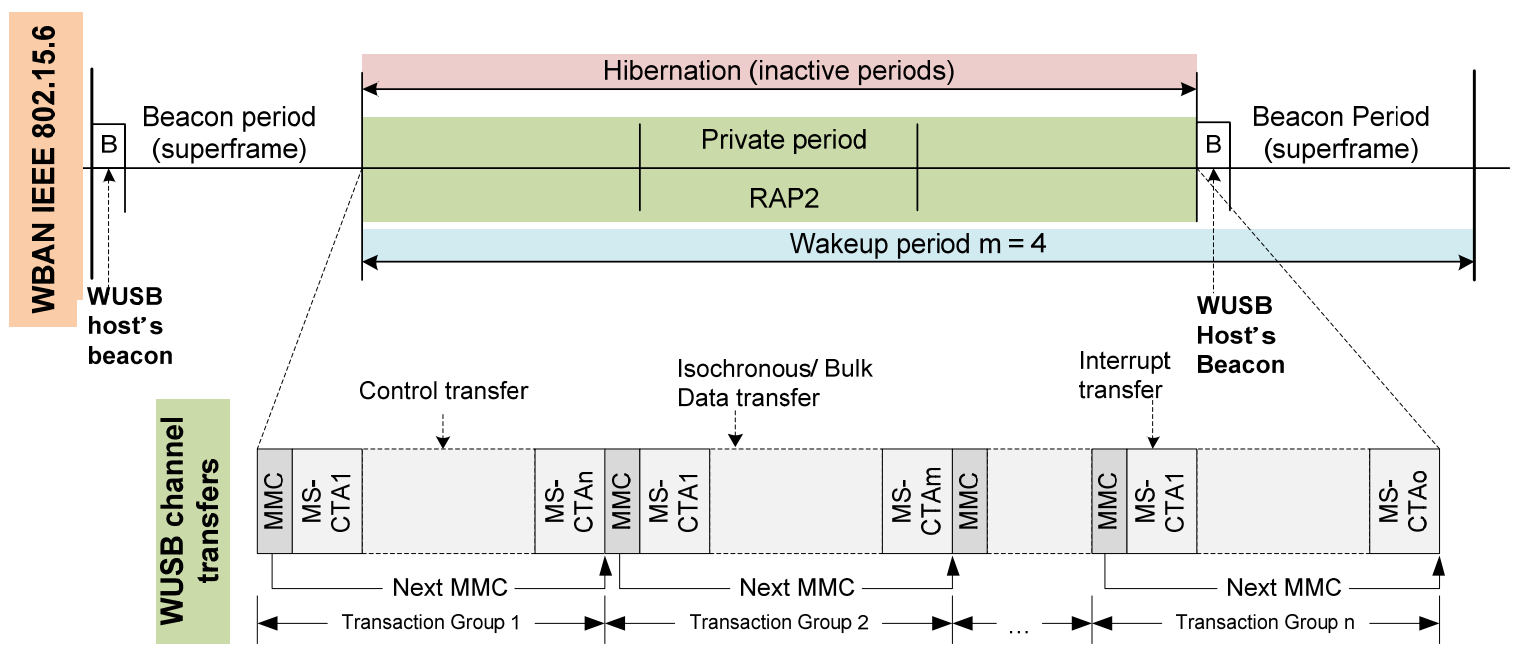

Fig. 4. Wireless universal serial bus (WUSB) private channel allocation at the $m$-periodic hibernation. WBAN: wireless body area network, RAP: random access phase, MMC: micro-scheduled management command, MS-CTA: micro-scheduled channel time allocation.

RAP2, Type-I/II access phase, and contention access phase (CAP) periods. The EAP1 and EAP2 periods are assigned through contention to data traffic with higher priorities. Further, the RAP1, RAP2, and CAP periods are assigned through contention to data traffic with lower priorities [5].

The IEEE 802.15.6 WBAN MAC systems have several MAC capability options. Fig. 2 shows current WBAN MAC Capability format standard. We denote the WUSB slave device that also performs the WBAN slave device function as a WUSB/WBAN slave device. The WUSB/WBAN slave device stays in its active mode during an entire superframe if the Always Active field is set to "one" in the received beacon in that superframe. Otherwise, if the Always Active field is set to "zero," the WUSB/WBAN slave device stays in its active mode during the beacon period and other allocated periods [5]. This operation is known as the hibernation.

The length of the hibernation in the IEEE 802.15.6 WBAN systems can be varied. If a WUSB/WBAN slave device wants to sleep during several superframes, it sets the Wakeup Period field in the Connection Request control frame and sends that frame to the WUSB/WBAN host as in Fig. 3. If the value of the Wakeup Period field is equal to $m$, it means that the slave device sleeps during $m-1$ superframes and goes into active mode at the $m$ th superframe. The Wakeup Phase field in the Connection Request control frame indicates the sequence number of the superframe where the device goes into active mode.

If the Wakeup Period field in the Connection Assignment control frame which the WUSB/WBAN host sends is set to a non-zero value, it means that the host assigned the $m$ periodic hibernation to its slave devices. Except for the RAP1 period, the length of the other periods can be set to zero. By using this feature, the WUSB/WBAN host allocates the WUSB private channels at the RAP2 period. Fig. 4 shows the WUSB private channel allocation scheme at the $m$-periodic hibernation of a WBAN superframe. At the $m$-periodic hibernation, there are $m-1$ inactive superframes. In this paper, an efficient WUSB private channel allocation method of LHT is proposed. In this method, the WUSB private channels are allocated during the inactive periods to improve channel utilization.

Fig. 5 shows the WUSB private channel allocation procedure for the $m$-periodic hibernation. When a request for WUSB data transmissions occurs in a WUSB cluster, the WUSB/WBAN host sets the WBAN beacon's RAP2 length field to the length of inactive periods required for $\mathrm{MMC}$ scheduling in the WUSB private channel. The WUSB/ WBAN host then transmits its beacon frame, and the Wakeup Period field in the Connection Assignment control frame is set to $m$ value. In addition, its Wakeup Phase field is set to a sequence number of the superframe increased by $m$. After receiving beacon and Connection Assignment control frames, non-WUSB WBAN slave devices enter into sleep mode during $m-1$ superframes. On the other hand, the WUSB/WBAN host and slave devices enter into active mode at every RAP2 period during consecutive $m$-1 superframes for WUSB transactions.

\section{RESULTS AND DISCUSSION}

Performance of the proposed scheme is evaluated through ns-2 simulations and WBAN PHY/MAC simulation parameters used in this paper [6-8]. The network size is $5 \mathrm{~m}$ $\times 5 \mathrm{~m}$. A maximum of 20 devices are randomly deployed into this area. The frame size is fixed to 4095 bytes. 


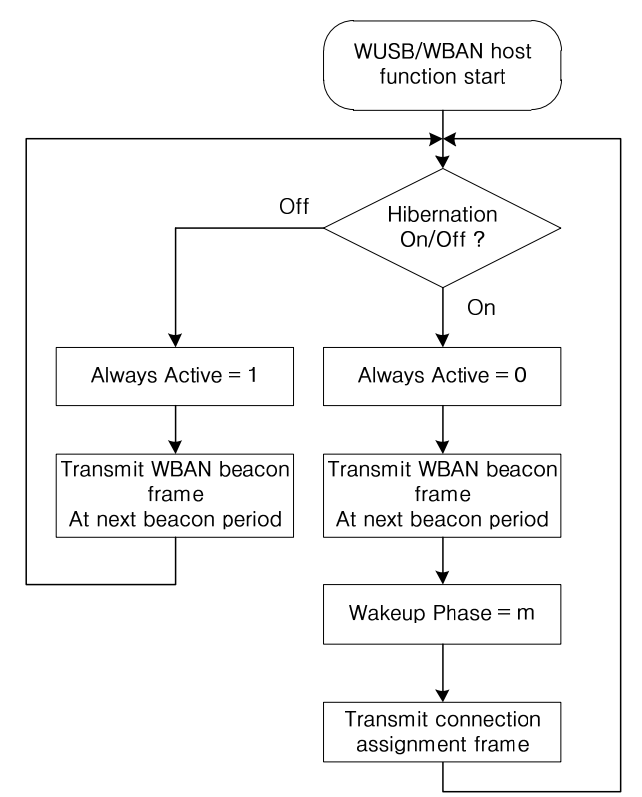

Fig. 5. Wireless universal serial bus (WUSB) private channel allocation at the $m$-periodic hibernation. WBAN: wireless body area network.

Fig. 6 shows the consumed energy per superframe of a WUSB/WBAN device according to the number of wakeup periods. In Fig. 6, the longer wakeup period reduces the consumed energy per WUSB/WBAN device except in the case where only one device exists. However, it increases with a larger number of WUSB/WBAN devices. This result is caused by more transmissions and receptions in a device due to the increased MMC scheduling overhead.

In the simulation for Fig. 7, there are four WBAN data streams between WUSB/WBAN host and its WBAN slave devices in a WBAN cluster.

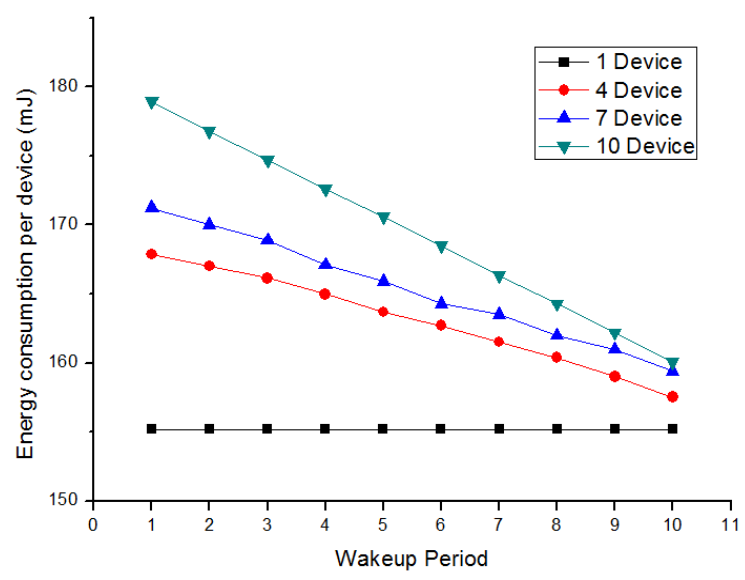

Fig.6. Energy consumption of a WUSB/WBAN device at each wakeup period. WUSB: wireless universal serial bus, WBAN: wireless body area network.

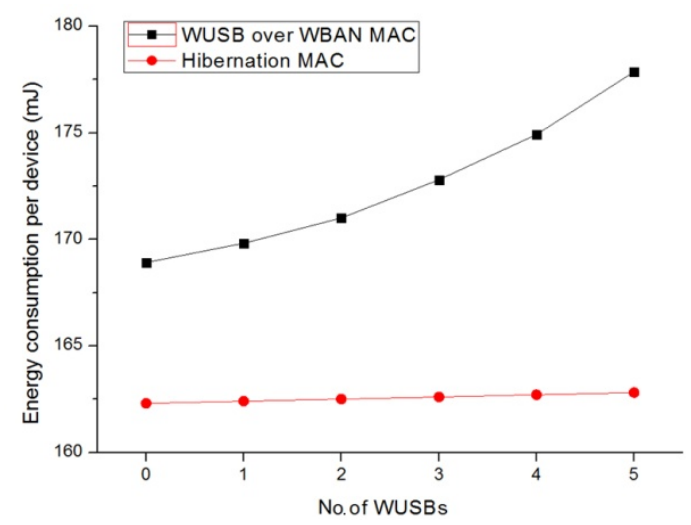

Fig. 7. Energy consumption of a non-WUSB WBAN device at each number of WUSB slave devices. WUSB: wireless universal serial bus, WBAN: wireless body area network, MAC: medium access control.

In that situation, each WUSB/WBAN device enters into that cluster and associates with the WUSB/WBAN host one by one. As the number of WUSB slave devices increases, the RAP2 periods allocated to WUSB channels become longer. Therefore, without LHT, this phenomenon leads non-WUSB WBAN devices to have longer scheduling delays and consume more energy. However, in LHT, the Wakeup Period is set to six WBAN superframes in the hibernation scheme. Then, the consumed energy of nonWUSB WBAN device does not change with up to five WUSB slave devices. This result is caused by the LHT mechanism in which the WUSB/WBAN host allocates the inactive periods for WUSB private channels by setting the beacon's RAP2 length field to the length of inactive periods while the other non-WUSB WBAN devices enter into sleep mode during five consecutive superframes.

\section{CONCLUSIONS}

In this paper, we integrated an IEEE 802.15.6 WBAN with WUSB system to develop wireless communication technologies for wireless wearable computer systems. In this paper, we proposed a LHT for WUSB over IEEE 802.15.6 hierarchical MAC to improve its energy efficiency. Simulation results show that the LHT also integrates WUSB transactions and WBAN traffic efficiently while it achieves high energy efficiency. The proposed LHT technique has compatibility with current IEEE 802.15.6 WBAN and WUSB standards.

\section{ACKNOWLEDGMENTS}

This research was supported in part by the Basic Science Research Program through the National Research Foundation 
of Korea (NRF) funded by the Ministry of Education, Science and Technology (MEST) (2010-0002366) and in part by the Mid-career Researcher Program through an NRF grant funded by the MEST (2011-0016145).

\section{REFERENCES}

[1] M. Patel and J. Wang, "Applications, challenges, and prospective in emerging body area networking technologies," IEEE Wireless Communications, vol. 17, no. 1, pp. 80-88, 2010.

[2] R. Palit, A. Singh, and K. Naik, "An architecture for enhancing capability and energy efficiency of wireless handheld devices," International Journal of Energy, Information and Communications, vol. 2, no. 4, pp. 117-136, 2011.

[3] USB Implementers Forum Inc., Wireless USB specification revision 1.1 [Internet], Available: http://www.usb.org/developers/wusb/docs.
[4] J. W. Kim, K. Hur, J. Park, and D. S. Eom, "A distributed MAC design for data collision-free wireless USB home networks," IEEE Transactions on Consumer Electronics, vol. 55, no. 3, pp. 1337$1343,2009$.

[5] IEEE, IEEE 802.15 WPAN task group 6 (TG6) body area networks [Internet], Available: http://www.ieee802.org/15/pub/TG6.html.

[6]. V. C. Nguyen, V. T. Pham, and B. K. Moon, "A new energy saving mechanism in IEEE 802.16e/m," International Journal of Energy, Information and Communications, vol. 2, no. 4, pp. 157-168, 2011.

[7] K. I. Kim, "Adjusting transmission power for real-time communications in wireless sensor networks." Journal of Information and Communication Convergence Engineering, vol. 10, no. 1, pp. 2126, 2012.

[8] M. Mana, M. Feham, and B. A. Bensaber, "SEKEBAN (secure and efficient key exchange for wireless body area network)." International Journal of Advanced Science and Technology, vol. 12, pp. 45-60, 2009.

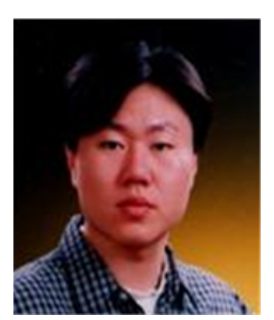

\section{Jounghoon Beh}

received the B.S., M.S., and Ph.D. degrees in the Department of Electronics Engineering from Korea University, Seoul, Korea in 2001, 2003, and 2008, respectively. He has been a Research Associate at University of Maryland Institute for Advanced Computer Studies (UMIACS), University of Maryland, College Park, MD, USA since January 2010. Previously he was a post-doctoral fellow in the School of Electronics Engineering, Korea University (from October 2008 to January 2010). His research interests are human robot interaction, pattern recognition, machine learning, and blind source separation.

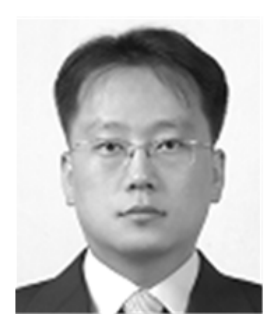

\section{Kyeong Hur}

is currently an Associate Professor in the Department of Computer Education at Gyeongin National University of Education, Republic of Korea. He was Senior Researcher with Samsung Advanced Institute of Technology (SAIT), Korea from September 2004 to August 2005. He received an M.S. and Ph.D. in the Department of Electronics and Computer Engineering from Korea University, Seoul, Korea, in 2000 and 2004, respectively. His research interests include computer network designs, next generation Internet, Internet QoS, and future All-IP networks.

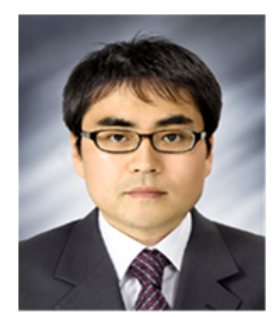

\section{Wooil Kim}

received the B.S., M.S., and Ph.D. degrees from the Department of Electronics Engineering from Korea University, Seoul, Korea in 1996, 1998, and 2003, respectively. He has been an Assistant Professor in the School of Computer Science and Engineering, University of Incheon, Incheon, Korea since August 2012. Previously, he was a Research Assistant Professor (from September 2007 to August 2012) and a Research Associate (from September 2005 to Augugst 2007) in the Erik Jonsson School of Engineering and Computer Science, University of Texas at Dallas, Richardson, TX, USA. He was also a post-doctoral researcher in the Electrical and Computer Engineering Department, Carnegie Mellon University, Pittsburgh, PA, USA (from September 2004 to August 2005) and Korea University (from September 2003 to August 2004). His research interests are human computer interfaces, human behavior signal processing, multimedia information retrieval, and pattern recognition.

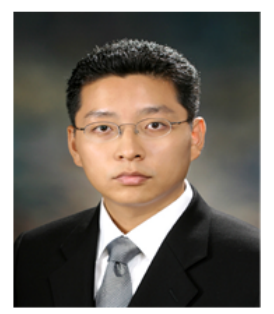

\section{Yang-Ick Joo}

is currently an Assistant Professor with the Division of Electrical and Electronics Engineering at Korea Maritime University, Busan, Korea, since March 2012. He received the M.S. and Ph.D. degrees from the Department of Electronics Engineering from Korea University, Seoul Korea, in 2000 and 2004, respectively. In addition, he was a Senior Engineer in the Division of Telecommunication Networks, Samsung Electronics, Suwon, Korea, from October 2004 to February 2012. His research interests include cross-layer solutions for wireless communication systems and resource allocation and management for WPAN and ubiquitous networking. 